\title{
A!
}

This is an electronic reprint of the original article.

This reprint may differ from the original in pagination and typographic detail.

Shariatmadari, Hamidreza; Li, Zexian; Uusitalo, Mikko A.; Iraji, Sassan; Jäntti, Riku

\section{Link adaptation design for ultra-reliable communications}

Published in:

2016 IEEE International Conference on Communications (ICC)

DOI:

10.1109/ICC.2016.7511429

Published: 01/05/2016

Document Version

Early version, also known as pre-print

Please cite the original version:

Shariatmadari, H., Li, Z., Uusitalo, M. A., Iraji, S., \& Jäntti, R. (2016). Link adaptation design for ultra-reliable communications. In 2016 IEEE International Conference on Communications (ICC) (pp. 1-5). (IEEE International Conference on Communications). IEEE. https://doi.org/10.1109/ICC.2016.7511429

This material is protected by copyright and other intellectual property rights, and duplication or sale of all or part of any of the repository collections is not permitted, except that material may be duplicated by you for your research use or educational purposes in electronic or print form. You must obtain permission for any other use. Electronic or print copies may not be offered, whether for sale or otherwise to anyone who is not an authorised user. 


\title{
Link Adaptation Design for Ultra-Reliable Communications
}

\author{
Hamidreza Shariatmadari*, Zexian $\mathrm{Li}^{\dagger}$, Mikko A. Uusitalo ${ }^{\dagger}$, Sassan Iraji*, and Riku Jäntti* \\ *Department of Communications and Networking, Aalto University, \{firstname.lastname\}@ aalto.fi \\ †Nokia Bell Labs, \{firstname.lastname\}@ nokia.com
}

\begin{abstract}
The fifth generation (5G) of cellular networks is expected to provide connectivity for a wide range of services. This requires the network to encounter novel features. Ultrareliable communications (URC) is one of the considered features, which provides a certain level of communication service almost all the time. This is essential in order to support mission-critical applications, such as industrial automation, public safety, and vehicular communications. This paper studies link adaptation optimization for URC, considering errors in both data and feedback channels. As the implementation of optimal link adaptation is challenging, particularly, for downlink transmissions due to the limited feedback channel, a simple link adaption scheme is also proposed. Results reveal that the performances of the proposed and optimal link adaptation schemes are close. Hence, the proposed scheme can be utilized to efficiently support URC in cellular networks.
\end{abstract}

Index Terms - Link adaptation, 5G, MCS, CQI, ultra-reliable communications, low-latency.

\section{INTRODUCTION}

The fifth generation (5G) of cellular systems is foreseen as an enabling technology for machine-type communications (MTC). The MTC applications can be utilized in various domains, including individual users and businesses. Providing efficient communications for MTC applications entails the network to support new features which are not deployed in the current cellular systems. The essential features for envisioned MTC applications include: support of massive number of machines, enhanced coverage, low power consumption, and ultra-reliable communications (URC) [1].

MTC enables a wide range of services and applications. The MTC applications have different requirements which make their deployments challenging in cellular systems. For instance, mission-critical applications, such as industrial automation, public safety, and vehicular communications, need data communication with high requirements on latency, reliability, and availability [2]. These indicate that the generated messages should be delivered with high success probability during a short period of time. For example, some industrial automation systems need high reliability corresponding to a maximum block error rate (BLER) of $10^{-9}$ with low latency up to 1 millisecond (ms) [3].

In order to enable URC in $5 \mathrm{G}$ systems, various network modifications and enhancements have been proposed [1]. For example, using shorter transmission time interval (TTI) reduces over-the-air transmission delay. To improve the link quality, it is suggested to utilize massive multiple-inputmultiple-output (MIMO) antennas, or provide relay nodes.
Supporting device-to-device (D2D) communications between nearby devices also allows reducing the delay by bypassing the data transmission through the cellular network [4]. New medium access control (MAC) schemes can be implemented for event-based applications to establish the communication link faster.

In wireless systems, link quality varies due to the path loss, fading, and interference. Adaptive modulation and coding (AMC) scheme is applied to adapt the data transmission to the link condition. AMC is performed by choosing an optimal combination of modulation and coding scheme (MCS) according to the link quality, fulfilling an error performance constraint [5]. In addition, the AMC is accompanied with error control schemes, such as automatic repeat request (ARQ) and hybrid ARQ (HARQ), in order to alleviate the occurred error in data transmissions. For instance, the employed AMC and HARQ schemes in long-term evolution (LTE) network allow achieving BLER of 1-10\% with end-to-end delay restricted to a few milliseconds [6]. Nevertheless, more accurate ACM schemes are essential to meet stringent URC requirements.

In this paper, we consider the AMC optimization for achieving very high reliability levels with low-latency. Generally, the delay constraint determines the maximum number of data transmission attempts for each payload. It is assumed that a payload can be delivered with maximum two transmission attempts, i.e., allowing one retransmission [7]. As the implementation of optimal AMC solution is challenging, in particular, for the downlink transmission, a simple AMC method is proposed. The proposed scheme enables performing the link adaptation with the limited feedback channel. Unlike most of the existing AMC schemes which take into account only error in the data channel, this paper considers errors occurred in both data and feedback channels.

The rest of the paper is organized as follows. Section II describes the system model and all the assumptions. Section III presents the optimal and proposed AMC schemes for URC operation. Section IV evaluates the performance of the AMC schemes applying to LTE physical layer framework, as an example. Finally, conclusions are drawn in Section V.

\section{SYSTEM MODEL}

Let us consider an URC system with the low-latency constraint over its communication link. The link is established between a base station and a user equipment (UE) for the uplink or downlink data transmissions. The problem in hand 
is to deliver each generated data payload within a short delay with high reliability. One way to tackle this problem is to use an error control scheme, such as automatic repeat request (ARQ) or hybrid ARQ (HARQ), to enhance the transmission efficiency[8]. What these methods do are retransmitting the payload which are received erroneously. However, the challenge is that the number of retransmission attempts for each payload is limited to one in order to meet the low-latency constraint in URC systems.

Since the quality of wireless link changes over time, the link adaptation is utilized by applying an AMC scheme. The base station is in charge of link adaptation by adjusting the employed coding rate and modulation type according to the channel conditions. In order to apply the link adaptation, the receiver needs to perform channel quality measurement. In LTE systems, the base station measures the sounding reference signal (SRS) received from the UE for the uplink channel estimation. For the downlink channel estimation, the UE measures the reference signal (RS) and estimates the quality of channel. The measured channel quality is delivered to the base station by the aid of channel quality indicator (CQI) values. Each CQI value is associated to a MCS corresponding to the highest possible rate that the UE can reliably decode.

Fig. 1 illustrates the signaling and data transmissions for uplink and downlink directions in the considered scenarios. It presents two payload transmissions. The first payload is delivered successfully with an initial transmission attempt. An acknowledgement (ACK) message informs the transmitter of successful reception. For the second payload, two transmission attempts are performed. This is due to the failure in the initial transmission attempt. A negative-acknowledgement (NACK) message is sent to the transmitter requesting the data retransmission. In addition, because TTI length is short in URC systems, channel is assumed to be block-flat fading [9]. Therefore, the channel conditions remain the same during all transmission attempts per each payload, while they change ergodically over time.

The above mentioned transmission policy requires utilizing data and control channels. The data channel is essential for conveying data payloads containing error detection information. The control channel delivers signaling information, including resource allocation, ACK, and NACK messages. In cellular systems, both data and control channels are prone to error due to the noise and interference in the system. The error in data channel mainly results in not decoding the payload data successfully. On the contrary, the error in control channel can arise various consequences. For instance, misinterpretation of ACK and NACK messages is one of the effects. Decoding the ACK as NACK results in performing unnecessary retransmission. On the other hand, decoding the NACK as ACK avoids performing the necessary transmission. Another issue, which is caused by error in control channel, is related to not decoding the resource allocation information correctly by a UE. In this case, the UE is not able to utilize the allocated resources for receiving or sending data. In LTE systems, different techniques are employed for control channel

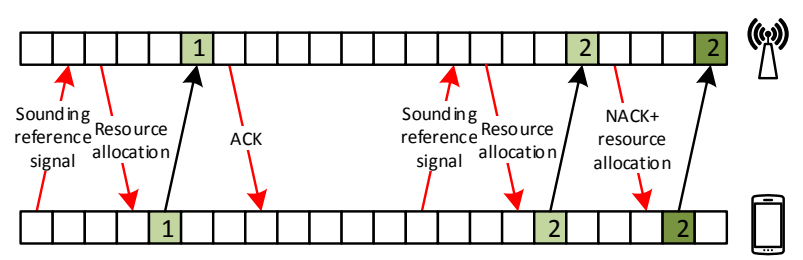

(a) Uplink transmission

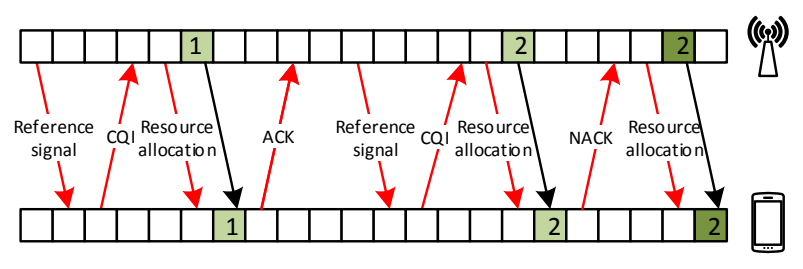

(b) Downlink transmission

Fig. 1: System model

to protect information and restrain the error. For instance, a high coding gain provides good robustness for the control information. For resource allocation messages, different levels of reliability are achievable by varying the control channel element (CCE) aggregation level [10]. In this way, it is possible to increase the probability of decoding resource assignment information successfully by allocating more resources.

In the development of AMC scheme for URC, it is assumed that the base station knows the exact or estimation of the error rate in decoding ACK/NACK messages. Furthermore, it is presumed that the error in decoding the resource allocation information is negligible. This can be achieved by employing a high aggregation level for CCE. Hence, the UE can properly determine and utilize the allocated radio resources.

The AMC aims at achieving the reliability target for the data transmissions. This is achievable by selecting an appropriate MCS from the predefined MCS set for each transmission attempt. Each MCS is characterized by its transmission rate and corresponding BLER. The transmission rate and BLER of the initial transmission attempt utilizing $M C S_{i}$ can be denoted as $r_{i}$ and $P_{1, i}(\gamma)$, where $\gamma$ denotes the received signal-tointerference-plus-noise ratio (SINR). The MCS set containing $M$ different MCSs can be ordered according to the offered rates and represented as $\left\{M C S_{1}, M C S_{2}, \ldots, M C S_{M}\right\}$.

The target reliability is considered with the residual BLER of $\epsilon$ after the retransmission attempt. This indicates the error in delivering each payload should be less than $\epsilon$ regardless of SINR level. In the next section, two different AMC schemes are proposed that are satisfying the reliability target.

\section{AMC FOR URC WITH LOW-LATENCY}

This section proposes two AMC schemes to guarantee the target reliability. The first scheme provides an optimal solution which maximizes the average throughput for each payload transmission. The second scheme is a sub-optimal solution that can be implemented easily, in particular, for downlink 
data transmissions.

\section{A. Optimal AMC scheme}

The optimal AMC scheme can be derived in order to maximize the average throughput for each payload transmission. This requires determining the appropriate MCSs for the transmission attempts, considering the reliability target, error in feedback channel, and the SNR level at the receiver. The expected throughput of a payload, allowing two transmission attempts, utilizing $m_{1}=M C S_{i}$ for the initial transmission and $m_{2}=M C S_{j}$ for the retransmission, can be formulated as [5]:

$$
\begin{aligned}
G= & r_{i}\left\{1-P_{1, i}(\gamma)\left(1-\xi_{A C K}\right)\right\}+ \\
& \frac{1}{\frac{1}{r_{i}}+\frac{1}{r_{j}}}\left\{\left(1-P_{1, i}(\gamma)\right) \xi_{A C K}\right)+ \\
& \left.P_{1, i}(\gamma)\left(1-\xi_{N A C K}\right)\left(1-P_{2, j}(\gamma)\right)\right\},
\end{aligned}
$$

where $\xi_{A C K}$ is the probability that ACK misinterpreted as NACK, $\xi_{N A C K}$ is the probability that NACK is decoded as ACK, $P_{1,1}(\gamma)$ is the residual BLER after the initial transmission attempt utilizing $M C S_{i}$, and $P_{2, j}(\gamma)$ is the residual BLER after the retransmission attempt utilizing $M C S_{j}$. Note that $P_{2, j}(\gamma)$ is independent from $P_{1, i}(\gamma)$ in case of utilizing ARQ scheme, i.e. $P_{2, j}(\gamma)=P_{1, j}(\gamma)$. In case of HARQ implementation, the residual BLER of the retransmission is conditionally dependent on the BLER of initial transmission; in this case, $P_{2, j}(\gamma) \leq P_{1, j}(\gamma)$.

The optimal MCSs for the initial and retransmission attempts can be found to maximize the expected throughput and satisfy the reliability target:

$$
\begin{aligned}
& m_{1}, m_{2}=\underset{1 \leq i, j \leq M}{\arg \max } G \\
& \text { subject to } \\
& 1-\left\{\left(1-P_{1, i}(\gamma)\right)+\right. \\
& \left.P_{1, i}(\gamma)\left(1-\xi_{N A C K}\right)\left(1-P_{2, j}(\gamma)\right)\right\} \leq \epsilon .
\end{aligned}
$$

It is worth mentioning that the optimal AMC scheme requires knowing the exact SINR value, in addition to the feedback error rates, i.e., $\xi_{A C K}$ and $\xi_{N A C K}$. This makes the implementation of optimal solution demanding for the downlink transmission. Because, the exact SINR value at the UE is not available for the base station which performs the AMC scheme. As mentioned earlier, the UE reports the estimated SINR value utilizing CQI values.

\section{B. Proposed AMC scheme}

As the base station is not able to determine the exact SINR value at the UE, due to the limited feedback channel, we propose a simple AMC scheme which is easily implementable for downlink data transmissions. In this method, the UE reports two CQI values for channel measurement feedback. The first CQI value determines the MCS for the initial transmission attempt and the second CQI value dictates the MCS for the case when the retransmission is required.
In LTE, the CQI values are mapped to MCSs considering $10 \%$ BLER performance. For a given SINR value, the highest CQI is reported which the corresponding MCS guarantees this target BLER [11]. The proposed scheme considers the similar approach; the base station determines two target BLERs and sends them to the UE. The UE utilizes these values to perform the SINR-to-CQI mapping and select two CQI values. The CQI values are then transmitted to the base station for performing the link adaptation.

We define a target BLER set as $E=\left\{\xi_{1}, \xi_{2}, \ldots, \xi_{r}\right\}$ whose components are arranged in decreasing order. The base station should select two BLER values form this set. It has been found that for achieving the maximum spectral efficiency when a payload is delivered within two transmission attempts, the initial transmission should be performed with a moderate BLER performance [12]. For instance, 10\% BLER for the initial transmission is favorable when the error in feedback channel is negligible. However, more reliable transmission is essential when the error in feedback channel is increased. In this scheme the preference is given to the initial transmission to perform the transmission with the highest possible BLER value. Then, the target BLER for the second transmission is determined as the highest possible value, considering the required reliability target. Algorithm 1 represents the selection of target BLERs, i.e. $e_{1}$ and $e_{2}$. Setting the smallest value of target BLER set $\left(\xi_{r}\right)$ smaller than the residual target BLER $(\epsilon)$ ensures that the algorithm finds the solution even when the error in feedback channel is high.

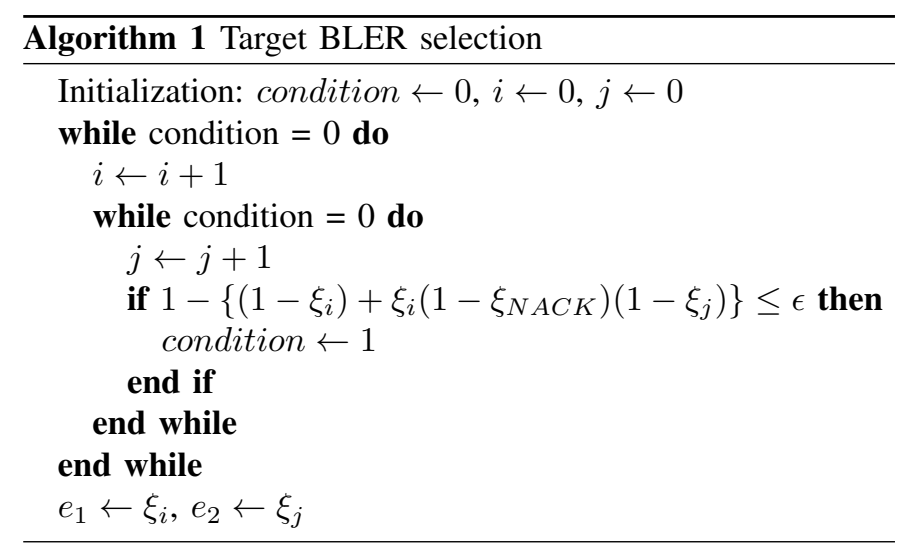

The base station delivers the selected target BLERs $\left(e_{1}\right.$ and $e_{2}$ ) to the UE. For each BLER, the UE needs to determine the highest CQI value so that its corresponding MCS guarantees that target. In case both target BLERs cannot be met with available MCSs, due to the low SINR level, the UE selects the CQI index corresponding to MCS with the lowest rate for transmissions. The MCS selection, $m_{1}$ and $m_{2}$, is performed as follows: 


$$
\begin{aligned}
& m_{1}(\gamma)= \begin{cases}M C S_{i} & \text { if } \exists i, j: P_{1, i}(\gamma) \leq e_{1}, P_{2, j}(\gamma) \leq e_{2} \\
M C S_{1} & \text { others }\end{cases} \\
& m_{2}(\gamma)= \begin{cases}M C S_{j} & \text { if } \exists i, j: P_{1, i}(\gamma) \leq e_{1}, P_{2, j}(\gamma) \leq e_{2} \\
M C S_{1} & \text { others }\end{cases}
\end{aligned}
$$

The UE then reports the selected CQI indexes to the base station. The base station performs resource allocation according to the received CQI values.

\section{Performance evaluations}

This section evaluates the performance of AMC schemes for providing URC. It compares the achieved throughputs for the proposed AMC scheme with the optimal solution. In addition, the minimum SINR level for achieving the target reliability is assessed. The performance analysis are performed by applying the AMC schemes to LTE physical layer framework. LTE defines 15 different CQI values driving different MCSs. The available MCSs are associated with coding rates between 1/13 to 1 , combined with 4-QAM, 16-QAM, and 64-QAM modulations [13]. For the proposed AMC scheme, we define $E=\left\{10^{-1}, 10^{-2}, \ldots, 10^{-10}\right\}$.

Fig. 2 represents the target BLERs for the initial and retransmission attempts employing ARQ scheme, for different reliability targets and various feedback channel errors. It is assumed that $\xi_{\text {feedback }}=\xi_{A C K}=\xi_{N A C K}$. For a reliable feedback channel, the performance target for the initial transmission is $10 \%$ BLER. However, the retransmission requires higher reliability depending on the target transmission reliability. When the error in feedback channel increases, more robust transmission is essential for the initial transmission. While, the retransmission is performed with a looser reliability performance.

Fig. 3 illustrates the expected throughput for the proposed and optimal schemes. In Fig. 3a, the considered transmission reliability corresponds to BLER of $10^{-5}$, while in Fig. 3b the considered target BLER for transmission is $10^{-10}$. It is evident that the performance of the proposed AMC scheme is very close to the optimal solution, in particular, when the feedback channel is error-free. The achieved throughout for the proposed AMC scheme is slightly lower than the optimal solution for some low SINR levels. This loss is originated from the fact that the UE cannot satisfactory find MCSs meeting the BLER targets; hence, it selects CQI index corresponding to the MCS with the lowest rate for both transmission attempts. In addition, the performance of the proposed scheme is reduced as the error in feedback channel increases.

Fig. 3 indicates the error in feedback channel results in lower achievable throughput. In addition, as the feedback channel error increases, the minimum SINR which guarantees the target reliability is also increased. For instance, data transmission with target BLER of $10^{-5}$ is achievable only for SINR levels greater than $-6 \mathrm{~dB}$ when the feedback channel is error-free; however, higher SINR is required when the error

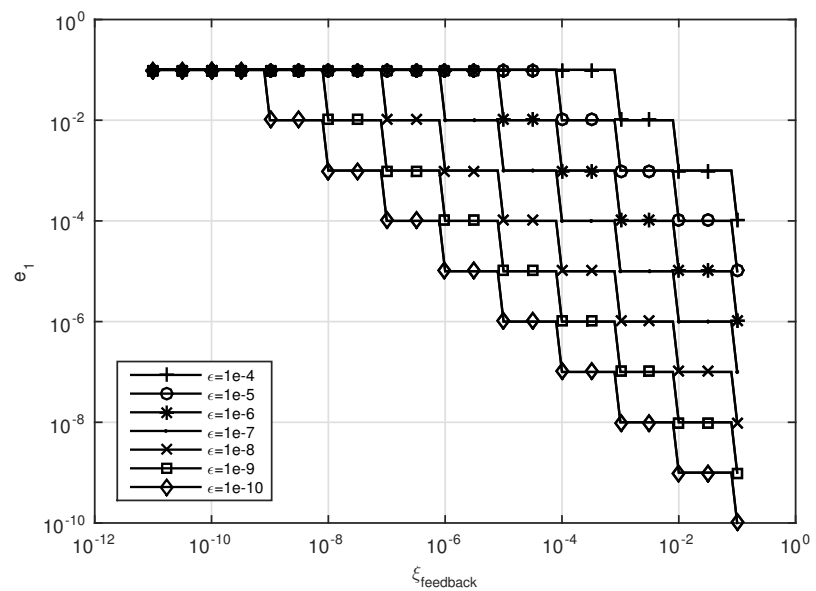

(a) Initial transmission

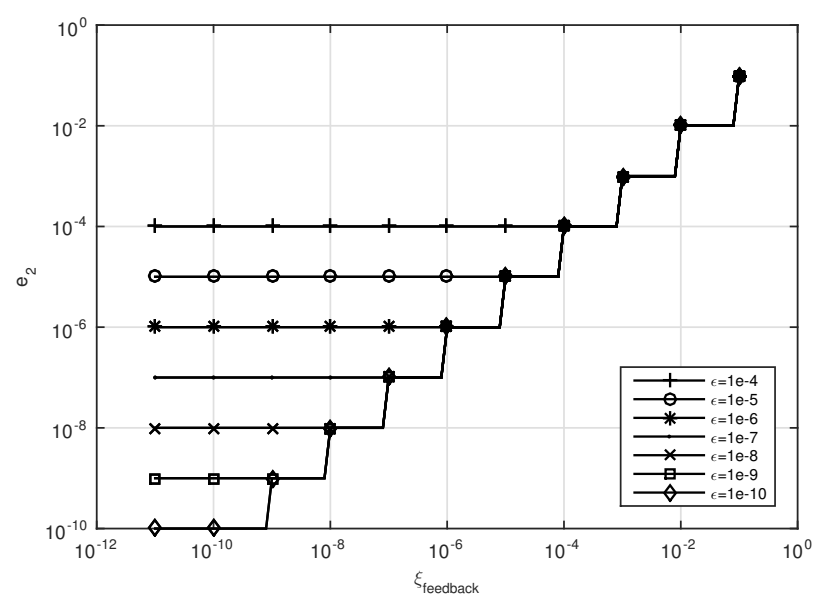

(b) Retransmission

Fig. 2: Target BLER for transmission attempts

occurs in feedback channel. The minimum SINR level for the proposed and optimal AMC schemes are the same, as both schemes choose the lowest MCS for both transmission attempts for low SINR levels. The lowest SINR level for a given reliability is achieved by using the MCS with the lowest rate and derived as:

$$
\begin{aligned}
& S I N R_{\text {min }}=\underset{\gamma}{\arg \min }\left\{1-\left\{\left(1-P_{1,1}(\gamma)\right)+\right.\right. \\
& \left.\left.P_{1,1}(\gamma)\left(1-\xi_{N A C K}\right)\left(1-P_{2,1}(\gamma)\right)\right\} \leq \epsilon\right\} .
\end{aligned}
$$

This indicates that the minimum SINR value depends on the target reliability and the error in decoding the NACK messages. Fig 4 illustrates the minimum SINR requirement for different target reliabilities and feedback channel errors. The higher target reliability and error in feedback channel increase the minimum required SINR level. It is evident that the minimum SINR level remains the same until the feedback error becomes more than 100 times of the BLER target. For instance, the minimum required SINR level achieving the BLER of $10^{-6}$ with feedback error rate of $10^{-4}$ is almost the same as for the error-free feedback channel. The minimum required SINR value is an important metric to determine 


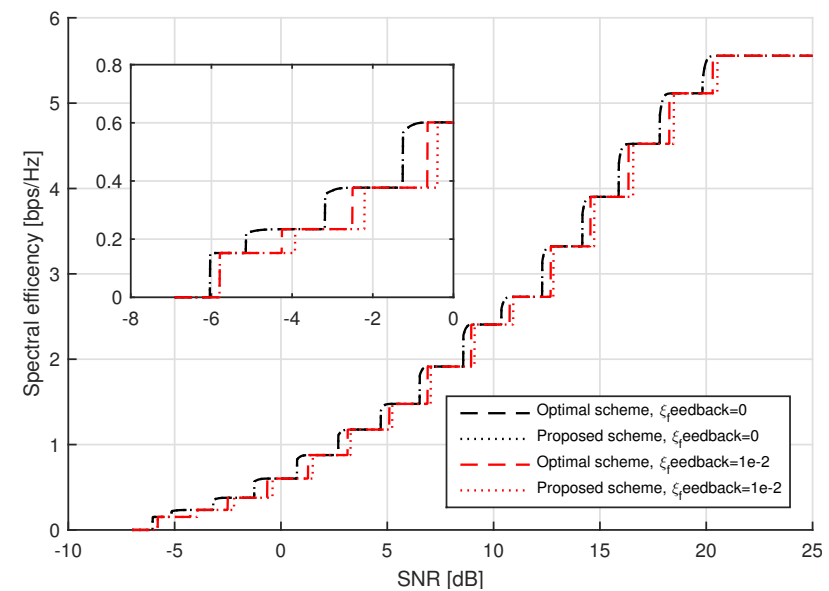

(a) $\epsilon=10^{-5}$

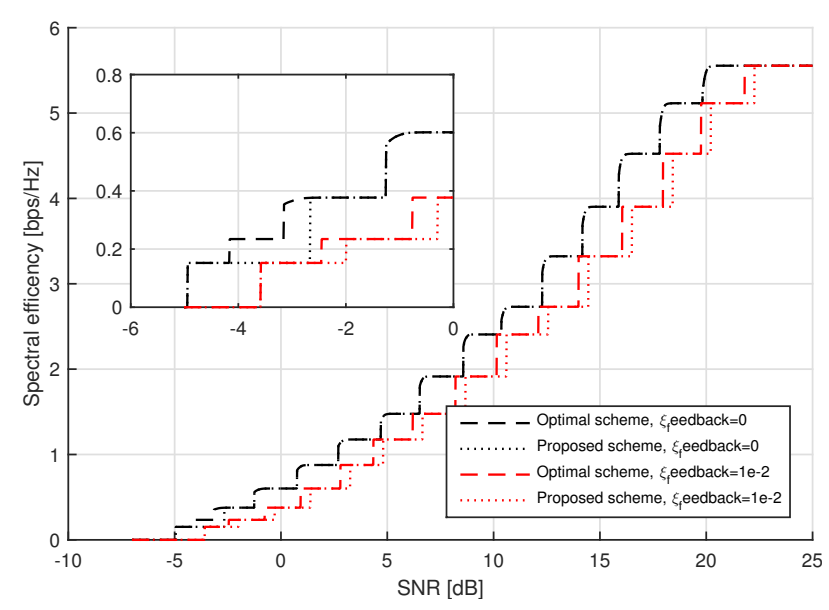

(b) $\epsilon=10^{-10}$

Fig. 3: Expected throughput for different reliability targets, considering error-free and error feedback channels.

the supported service range, particularly, for users located in the edge of the cell. It helps in designing the network and managing the interference in the cell for URC support [14].

\section{Conclusions}

In this paper, we have considered the link adaptation designs for URC with low latency. The optimal AMC is derived to achieve the maximum throughput for each payload transmission. A sub-optimal AMC scheme is proposed which can be implemented with limited feedback channel. The performance of the proposed scheme is close to the optimal solution, hence it can be implemented as downlink ACM design in the future wireless systems, e.g., 5G systems. In addition, the paper assessed the effects of feedback channel error in terms of achieved throughput and the minimum required SINR level supporting the desired reliability level.

\section{REFERENCES}

[1] H. Shariatmadari, R. Ratasuk, S. Iraji, A. Laya, T. Taleb, R. Jäntti, and A. Ghosh, "Machine-type communications: current status and future perspectives toward 5G systems," IEEE Communications Magazine, vol. 53, no. 9, pp. 10-17, sep 2015.

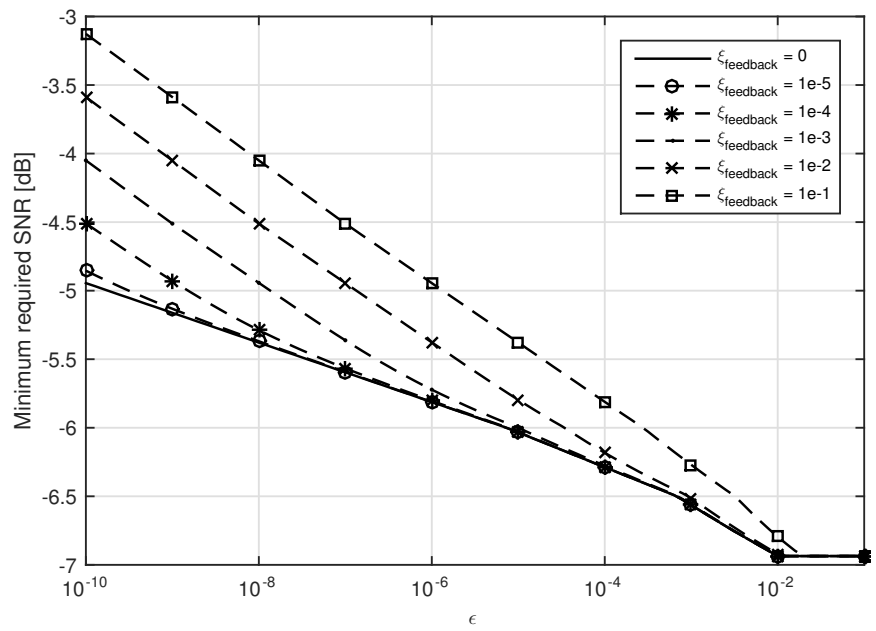

Fig. 4: Minimum SNR level for supporting the target reliability considering the feedback channel error.

[2] N. A. Johansson, Y.-P. E. Wang, E. Eriksson, and M. Hessler, "Radio access for ultra-reliable and low-latency 5G communications," in 2015 IEEE International Conference on Communication Workshop (ICCW), jun 2015, pp. 1184-1189. [Online]. Available: http://ieeexplore.ieee.org/lpdocs/epic03/wrapper.htm?arnumber=7247338

[3] E. Dahlman, G. Mildh, S. Parkvall, J. Peisa, J. Sachs, Y. Selen, and J. Skold, "5G wireless access: requirements and realization," IEEE Communications Magazine, vol. 52, no. 12, pp. 42-47, dec 2014.

[4] H. Shariatmadari, S. Iraji, and R. Jäntti, "Delay Analysis of Network Architectures for Machine-to-Machine Communications in LTE System," in IEEE International Conference on Telecommunications (ICT), Workshop on M2M Solutions and Services, 2014.

[5] C.-H. Yu, A. Hellsten, and O. Tirkkonen, "Rate Adaptation of AMC/HARQ Systems with CQI Errors," in 2010 IEEE 71st Vehicular Technology Conference (VTC 2010-Spring), may 2010, pp. 1-5.

[6] S. Parkvall, A. Furuskar, and E. Dahlman, "Evolution of LTE toward IMT-advanced," IEEE Communications Magazine, vol. 49, no. 2, pp. 84-91, feb 2011.

[7] A. Osseiran, F. Boccardi, V. Braun, K. Kusume, P. Marsch, M. Maternia, O. Queseth, M. Schellmann, H. Schotten, H. Taoka, H. Tullberg, M. a. Uusitalo, B. Timus, and M. Fallgren, "Scenarios for 5G mobile and wireless communications: the vision of the METIS project," IEEE Communications Magazine, vol. 52, no. 5, pp. 26-35, may 2014. [Online]. Available: http://ieeexplore.ieee.org/lpdocs/epic03/wrapper.htm?arnumber=6815890

[8] H. Shariatmadari, A. Mahmood, and R. Jantti, "Channel ranking based on packet delivery ratio estimation in wireless sensor networks," in 2013 IEEE Wireless Communications and Networking Conference (WCNC), apr 2013, pp. 59-64.

[9] P. Agyapong, M. Iwamura, D. Staehle, W. Kiess, and A. Benjebbour, "Design considerations for a 5G network architecture," IEEE Соттиnications Magazine, vol. 52, no. 11, pp. 65-75, nov 2014.

[10] M. Wu, Y. Peng, X. Yang, X. Zhang, and W. Wang, "Performance analysis of physical downlink and uplink channels in TD-LTE system," in Communication Technology (ICCT), 2010 12th IEEE International Conference on, nov 2010, pp. 308-311.

[11] J. C. Ikuno, M. Wrulich, and M. Rupp, "System Level Simulation of LTE Networks," in IEEE 71st Vehicular Technology Conference (VTC 2010-Spring), 2010, may 2010, pp. 1-5.

[12] H. Shariatmadari, S. Iraji, and R. Jäntti, "Analysis of Transmission Methods for Ultra-Reliable Communications," in Proc. IEEE Personal, Indoor and Mobile Radio Communications (PIMRC) Workshop on M2M Communications: Challenges, Solutions and Applications, 2015, pp. 1126-1131.

[13] TS136.213, "E-UTRA; physical layer procedures," Tech. Rep., 2013.

[14] N. Saquib, E. Hossain, and D. I. Kim, "Fractional frequency reuse for interference management in LTE-advanced hetnets," IEEE Wireless Communications, vol. 20, no. 2, pp. 113-122, apr 2013. 